\title{
Objective evaluation of blood flow in the small-intestinal villous: quantification of findings from dynamic endoscopy with concomitant narrow-band imaging $\square$
}

\section{(이요 $\odot$}

\author{
Authors \\ Institutions \\ 1 Department of Gastroenterology and Hepatology, \\ Nagoya University Graduate School of Medicine, \\ Nagoya, Aichi, Japan \\ 2 Department of Endoscopy, Nagoya University Hospital, \\ Nagoya, Aichi, Japan \\ 3 Department of Internal Medicine, Division of \\ Therapeutic Medicine, Nagoya University Graduate \\ School of Medicine, Nagoya, Aichi, Japan
}

Tomoaki Takeyama1', Yoshiki Hirooka², Hiroki Kawashima', Eizaburo Ohno¹, Takuya Ishikawa1, Takeshi Yamamura', Kazuhiro Furukawa' ${ }^{1}$, Kohei Funasaka², Masanao Nakamura ${ }^{3}$, Ryoji Miyahara' ${ }^{1}$, Masatoshi ishigami ${ }^{1}$, Hidemi Goto ${ }^{1}$

submitted 23.11.2017

accepted after revision 9.4.2018

\section{Bibliography}

DOI https://doi.org/10.1055/a-0619-4965 |

Endoscopy International Open 2018; 06: E941-E949

(C) Georg Thieme Verlag KG Stuttgart · New York

ISSN 2364-3722

Corresponding author

Yoshiki Hirooka, Department of Endoscopy, Nagoya

University Hospital, 65, Tsuruma-Cho, Showa-Ku, Nagoya,

Aichi 466-8550, Japan

Fax: +81-52-7358806

hirooka@med.nagoya-u.ac.jp

\section{ABSTRACT}

Background and aims We have previously shown that the increase in blood flow volume in jejunum villi after spraying of $10 \%$ dextrose solution correlates with pancreatic exo- crine function (PEF). The aim of this study was to establish an objective method to evaluate the amount of jejunum villous blood flow using a novel image analysis system.

Patients and methods The subjects were 26 patients who underwent upper gastrointestinal endoscopy with a newly developed small intestine endoscope (SIF-Y0007, Olympus, Tokyo, Japan). By defining the ratio of capillary occupancy in each villus at levels from 1 to 5 , villous blood flow was evaluated subjectively on the villous blood flow scale (VBFS). Objective evaluation was performed based on luminance analysis. The morphological opening process was used to make images with leveled brightness. A histogram was prepared from the luminance information and the standard deviation was determined and defined as SDOV (Standard Deviation calculated from a histogram made by luminance analysis Of Villi). PEF was evaluated by measuring the BT-PABA (N-benzoyl-L-tyrosyl-p-aminobenzoic acid) excretion rate.

Results There was a significant positive correlation between VBFS and SDOV $(P<0.0001, \rho=0.5882)$. SDOV was also positively correlated with PEF $(P=0.0004, \rho=0.6421)$.

Conclusions SDOV is a new objective index for evaluation of blood flow volume in jejunum villi. SDOV may be useful in clinical practice to estimate PEF and for clarification of the mechanisms underlying the functional correlation between the pancreas and small intestine.

University Hospital Medical Network Clinical Trials Registry UMIN000014200

TRIAL REGISTRATION: Retrospective, Observational SingleCenter Study UMIN000014200 at http://www.umin.ac.jp

\section{Introduction}

Insufficiency of pancreas exocrine function leads to malnutrition and causes a worse prognosis in patients with chronic pancreatitis or pancreatic cancer. Therefore, it is important to understand the mechanism of malnutrition, especially in patients with pancreatic disease $[1,2]$. The pancreas and small intestine play central roles in digestion and absorption of nutrients, and a close interaction between these organs is likely. For example, pancreatic juice containing digestive enzymes is considered to be an important factor for small intestinal villous growth [3,4]. However, since an endoscopic approach to the small intestine is anatomically difficult, there are only a few studies on the interaction of these organs, and most interactions in humans have not been defined $[4,5]$. 
A prototype endoscope for the small intestine (SIF-Y0002, Olympus, Tokyo, Japan) can be used to observe the proximal jejunum without use of a balloon, and allows morphological evaluation of villi by biopsy [6]. This approach has shown significant atrophy in small-intestinal villi in patients with chronic pancreatitis with reduced pancreatic exocrine function (PEF); the villus form was closely correlated with PEF; and incretin-secreting cells, which are also likely to be correlated with PEF, were found to be expressed in the upper small intestine [6].

Using a recently developed small intestine endoscope (SIFY0007, Olympus, Tokyo, Japan) with $80 \times$ magnification power for optical zooming, the capillary network and blood flow in each villus can be observed in magnified observation with concomitant use of narrow-band imaging (NBI) ( $>$ Video 1 ). In addition, we found that the blood flow volume in villi increased when $10 \%$ dextrose solution was applied to the small intestine mucosa (thicker blood vessels and a deeper brown color in the image were seen in magnified observation with NBI). To clarify the relationship between this phenomenon and small-intestinal mucosal function, subjective changes in blood flow (changes in color) before and after spraying dextrose solution were examined in animated observation of endoscopic findings, using an ascending scale of 1 to 5 . This was defined as the villous blood flow change. That study showed that villous atrophy had a negative correlation with villous blood flow change and a positive correlation with the PABA excretion rate in urine, an index of $\mathrm{PEF}$ (that is, an increase in blood flow was correlated with PEF) [7].

These data indicate that PEF can be measured using magnifying endoscopy with concomitant use of NBI in small-intestinal mucosa. However, villous blood flow change is based on subjective evaluation, and is not an objective index. Therefore, the current study was performed to develop a method to quantify blood flow in small-intestinal villi objectively, based on the depth of brown color in endoscopic findings after processing of images obtained using magnified observation with NBI.

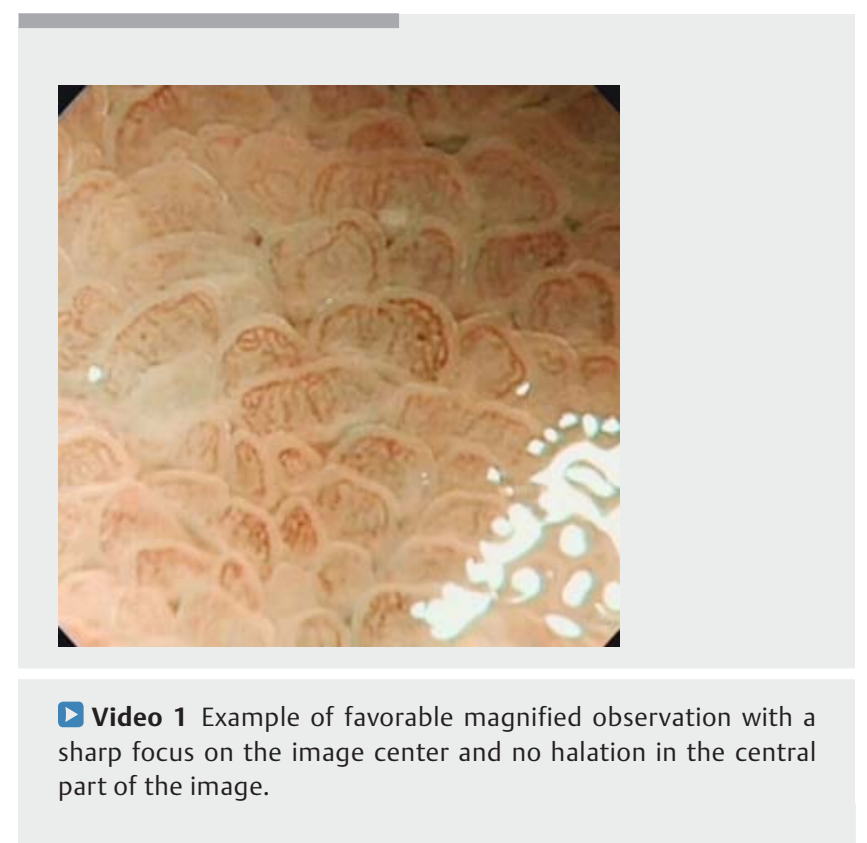

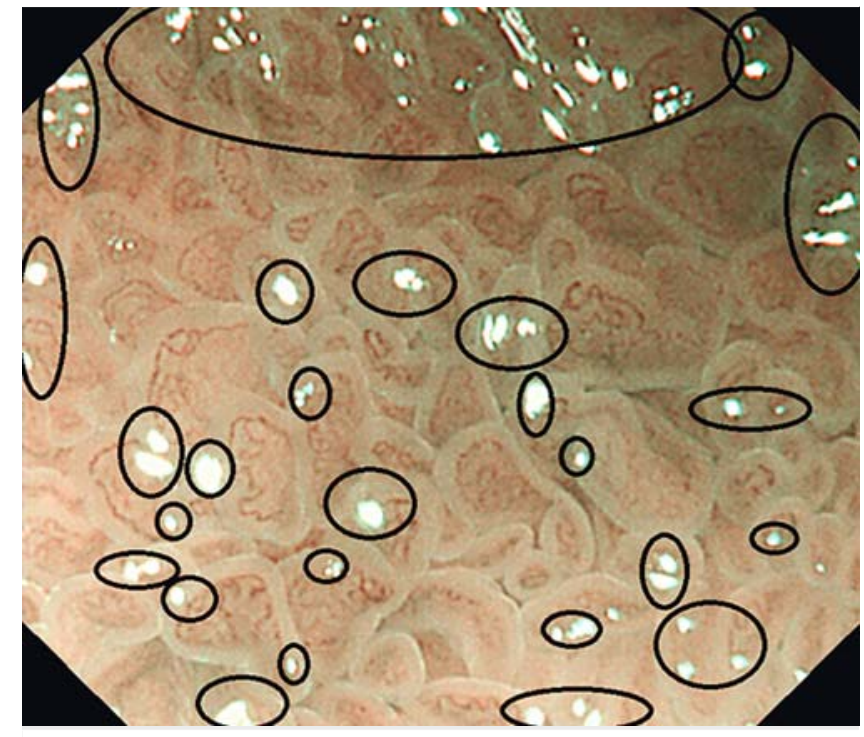

- Fig. 1 Example of halation (circles). Halation is a phenomenon that causes whiteout of an image because of the reflection of light from the endoscope.

\section{Patients and methods}

\section{Study design}

This was a retrospective, observational, open label, single center, phase I study.

\section{Subjects}

Among 30 patients who underwent small intestinal endoscopy with a prototype small intestinal endoscope with a magnifying function (SIF-Y0007, Olympus) from August 2015 to September 2016, 26 who could be favorably followed by magnified observation with concomitant NBI were selected as the subjects. The inclusion criteria were patients with pancreatic disease such as pancreatitis and pancreatic cancer or patients without pancreatic disease as controls and adults aged $\geq 20$ years who gave informed consent to the study and who required upper gastrointestinal tract endoscopy for screening for upper gastrointestinal disease (e.g. gastric cancer or gastric ulcer). The exclusion criteria included premenopausal women, patients with severe malnutrition, a history of surgery for the stomach/ duodenum/small intestine, internal administration of antiplatelet drugs or anticoagulants, routine use of NSAIDs, severe chronic cardiac failure/chronic renal failure, and no provision of written informed consent.

Favorable magnified observation was defined as continuous video uptake in a 3-minute interval after spraying dextrose solution with a sharp focus on the image center and no halation ( $\triangleright$ Fig. 1) in the central part of the image taken at the highest magnification (see $>$ Video 1: example of favorable observation; and - Video 2: example of inappropriate observation because of halation). Subjects with autoimmune or chronic pancreatitis were diagnosed based on ICDC2011 [8,9], and those with pancreatic cancer were histopathologically diagnosed with pancreatic duct cancer by surgery or endoscopic ultra- 
sound (EUS)-guided fine needle aspiration. Subjects with nonpancreatic disease included those with unexplained abdominal pain, abdominal symptoms such as diarrhea, and a need to receive an endoscopic examination of the upper digestive tract for preoperative screening for conditions such as gallstones. In all subjects, the absence of pancreatic disease was shown based on the results of blood tests, abdominal ultrasonography, and computed tomography (CT).

\section{Data collection}

Data for sex, date of birth, age, height, and weight were collected for all patients. Blood tests (red blood cell count, hemoglobin level, white blood cell count, platelet count) and biochemical tests (GOT, GPT, ALP, yGTP, LDH, total bilirubin, AMY, lipase, elastase 1, total protein, albumin, prealbumin (TTR), retinol-binding protein (RBP), vitamin D3 (VitD3), Fe, UIBC, Zn, Mg, ferritin, transferrin, total cholesterol level, LDL cholesterol level, HDL cholesterol level, triglyceride, blood glucose level, HbA1c, INS, C-peptide, IgG, IgA, IgM, IgE, BUN, Cr, Na, Cl, K, Ca, PT, APTT) were performed in all patients. All data were collected retrospectively.

\section{Pancreatic exocrine function (PEF) test}

PEF was evaluated by measuring the PT-PABA excretion rate. This was measured twice and the mean value was used as the PEF value.

\section{Endoscopic examination}

The procedure was performed with an improved enteroscope with $80 \times$ magnification function in optical zooming (SIFY0007: working length $2000 \mathrm{~mm}$, outer diameter $9.2 \mathrm{~mm}$, and channel size $2.8 \mathrm{~mm}$; Olympus), which can reach the jejunum without balloon assistance. The endoscopy video system was an Evis Lucera Spectrum (CV-260/CLV-260SL) (Olympus) and the NBI image was created using the sequential scanning method. Using the SIF-Y0007, push enteroscopy was performed with the oral method without any overtube or balloon assistance,

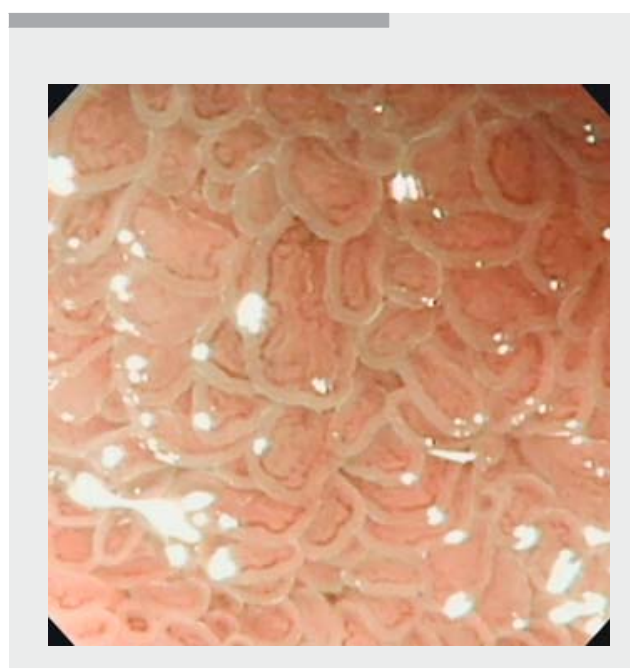

$\checkmark$ Video 2 Example of inappropriate magnified observation because of halation.

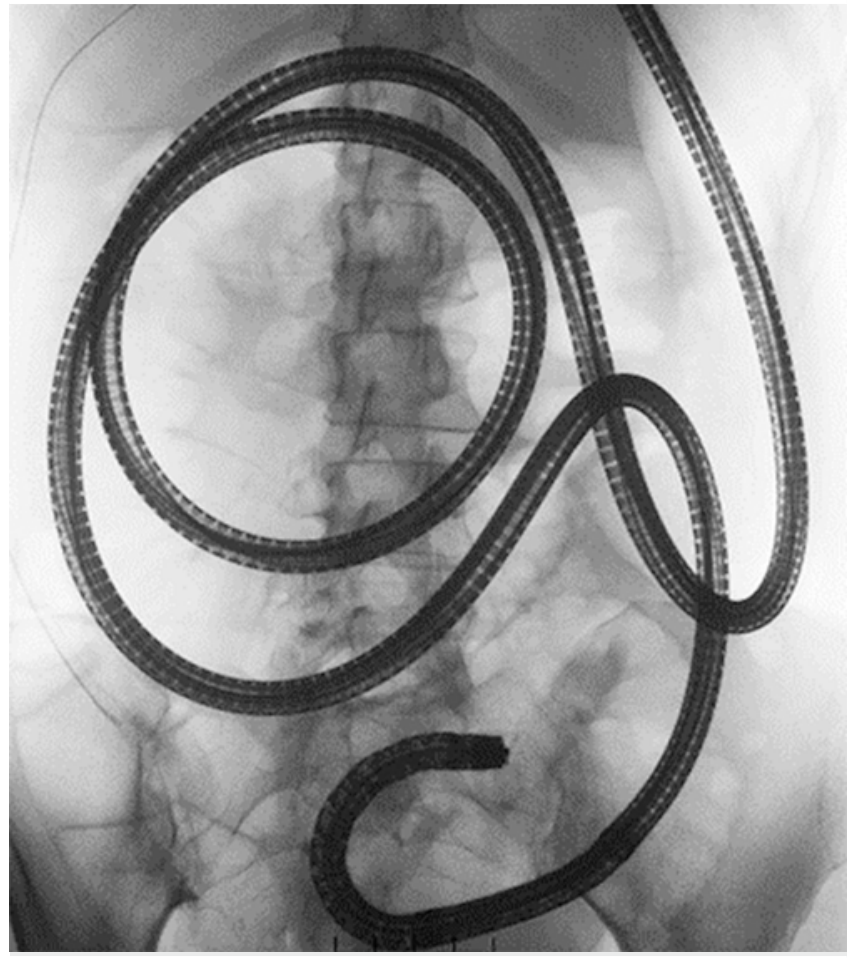

- Fig.2 Insertion to the jejunum was confirmed by fluoroscopy.

with sedation by intravenous injection of $15 \mathrm{mg}$ midazolam under appropriate monitoring. After observation of the upper gastrointestinal such as the esophagus, stomach, and duodenum, air in the stomach was absorbed to insert the endoscope to the proximal jejunum. The duodenojejunal flexure was identified using fluoroscopic findings in all cases and the position of Treitz ligament was determined. At first, the scope was inserted as deeply as possible. Next, the depth when the tip had begun to withdraw was determined by pulling back the endoscope. We then determined the depth when the tip was withdrawn to Treitz ligament. The difference in these two measurements was defined as the insertion depth beyond Treitz ligament. We aimed to reach at least $60 \mathrm{~cm}$ beyond Treitz ligament but not

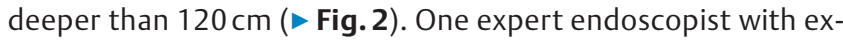
perience of about 300 cases using an SIF-Y0007 performed all of the procedures.

\section{Observation of the jejunum}

After confirming the approach to the proximal jejunum under fluoroscopic guidance, observation was started with concomitant $\mathrm{NBI}$ at maximum magnification. After spraying $20 \mathrm{~mL}$ of $10 \%$ dextrose solution, changes in villous blood flow were observed for about 3 minutes. Observation was performed for regions with no reddening caused by contact of the endoscope. Videos were recorded and photographs were taken about every 30 seconds during the observation. 


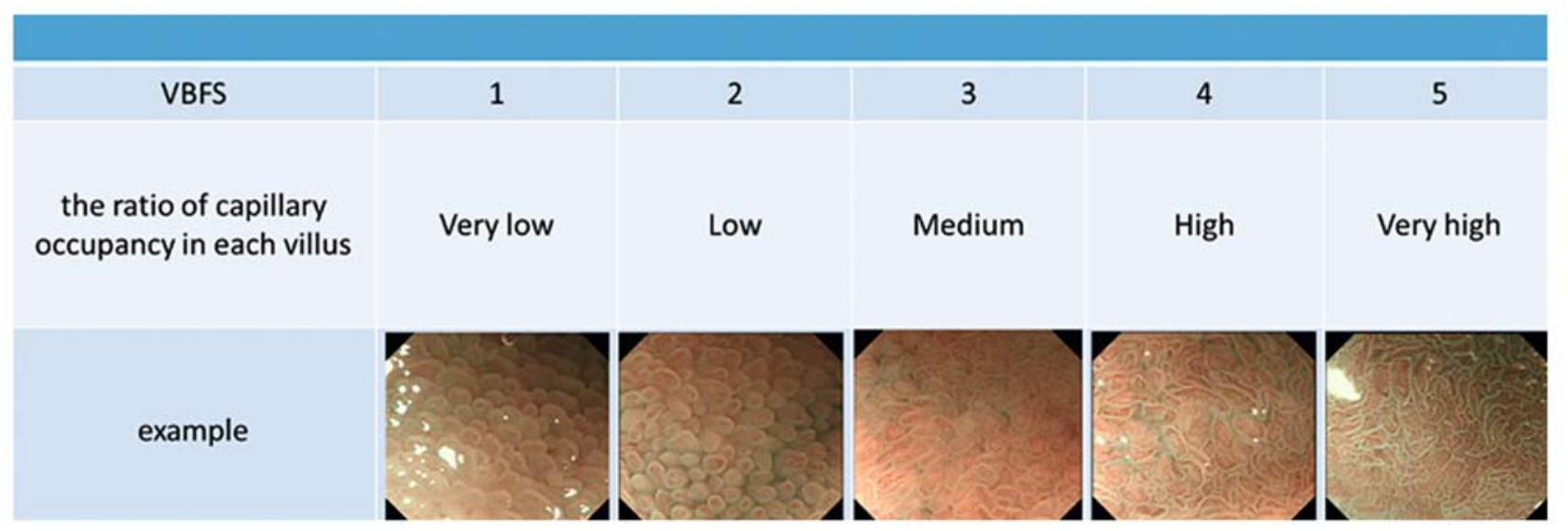

Fig. 3 Villous blood flow scale (VBFS). Two endoscopists evaluated the ratio of capillary occupancy in each villus at levels from 1 to 5 in ascending order regardless of color tone. We defined the smaller number of the two values as VBFS.

\section{Subjective evaluation of villous blood flow in the small intestine using still images: VBFS}

Since there is no established criterion or index for evaluation of villous flow volume using an endoscopic image, the evaluation was made by two endoscopists using a five-point scale method ( $>$ Fig.3). The two endoscopists evaluated the ratio of capillary occupancy in each villus at levels from 1 to 5 in ascending order regardless of color tone, and defined this as the villous blood flow scale (VBFS). An intra- and interobserver variability analysis for VBFS gave $\mathrm{k}$ coefficients of 0.2324 and 0.2018 , respectively. The maximum VBFS in 3 minutes after spraying dextrose solution was compared with PEF.

\section{Objective evaluation of villous blood flow in the small intestine using still images: SDOV}

Only subjective evaluation was performed for VBFS, and thus we aimed to analyze the villous blood flow automatically and objectively based on luminance information, using computer analysis.

However, there are several problems to solve for appropriate luminance analysis (see $>$ Fig. 4). First, since a sharp focus is visually adjusted to the center of an image in an endoscopic examination, the reliability of the luminance value is low in the peripheral part of the image ( $\mathbf{F i g . 4 a )}$. Second, since lights were located at the lower left in a SIF-Y0007 image, the brightness was not consistent across the image ( $\nabla$ Fig. $\mathbf{4 b}$ ). When the brightness varies, the luminance also varies, and so we had to standardize the brightness of each villus before measurement of the luminance. Third, when there is halation in the image, the luminance value gives a much higher value than the actual value ( $>$ Fig. $4 \mathbf{c}$ ).

For these reasons, we performed several processes before carrying out luminance analysis (see Figs.5-7). First, we used $1 / 4$ of the image center as the region of interest (ROI) because the peripheral part of the endoscopic image was blurred and resulted in a luminance value with low reliability ( $>$ Fig. 5a).
Second, the brightness of each villus was standardized using MATLAB's morphological operation $[10,11]$ (the full details are in $>$ Fig. $\mathbf{5 b}$ and $>$ Fig. 5c). Lastly, luminance values of all of the pixels were determined and a histogram of these data was created. To exclude halation, pixels whose luminance was within the range 0 - 99 were used to determine the standard deviation (SD) and defined as SDOV (Standard Deviation calculated from a histogram made by luminance analysis Of Villi).

When blood flow increases in the villi, the number of pixels with low luminance will increase and show large variations. This results in increased SDOV ( $\triangleright$ Fig. 7). All image analyses were performed using MATLAB and Image Processing Toolbox (MATLAB and Image Processing Toolbox R2015b, The MathWorks Inc., Natick, MA, USA).

\section{Statistical analysis}

First, we compared VBFS and SDOV in 268 NBI magnified images obtained from all patients. Then VBFS and SDOV were assessed in $6 \mathrm{NBI}$ magnified images taken at intervals of about 30 seconds in the 3-minute interval after spraying $20 \mathrm{~mL}$ of $10 \%$ dextrose solution. The maximum values of these indexes were compared with PEF (PT-PABA excretion rate). In all analyses, correlations were examined using the Spearman rank method in R3.3.0 [12] (available via http://www.R-project.org) and ggplot2 [13].

\section{Ethics}

This study was performed after obtaining approval from the Ethics Review Committee of Nagoya University Hospital. Written informed consent was obtained from the patients after they were provided with an explanation of the study (UMINCTR No.000014200, IRB:2014-0064). 

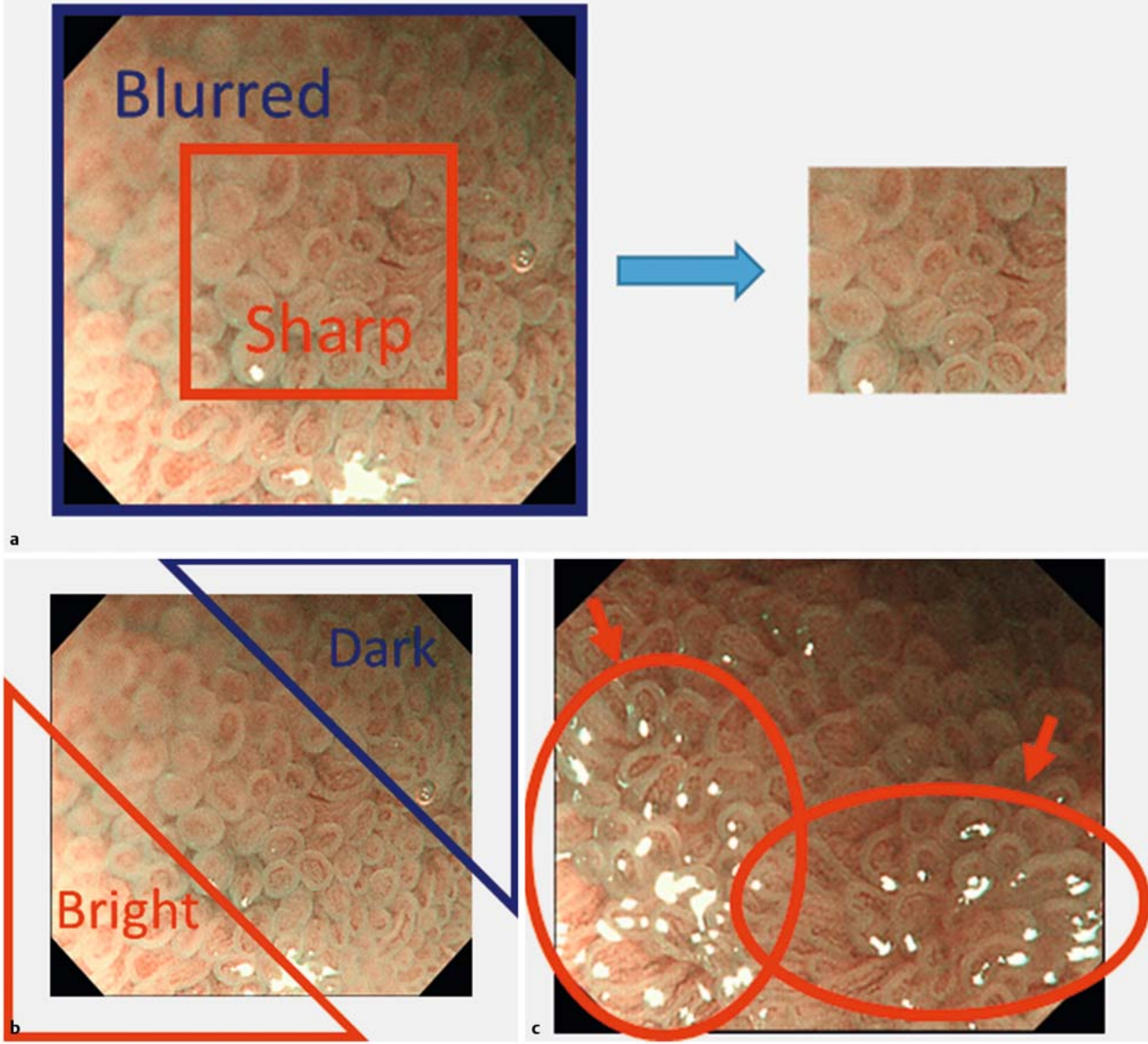

- Fig. 4 a The peripheral part of the endoscopic image is blurred and results in a luminance value with low reliability. Therefore we used $1 / 4$ of the image center as the region of interest (ROI). $\mathbf{b}$ The brightness was not consistent across the image. Therefore we had to standardize the brightness of each villus. c Halation (arrows) affects the luminance information since its luminance value is much higher than the actual value.

\section{Results}

\section{Patients}

The target diseases were chronic pancreatitis ( $n=13$ subjects), autoimmune pancreatitis $(n=5)$, and pancreatic cancer $(n=1)$. Based on the results of blood tests, abdominal ultrasonography, and $C T$, patients without pancreatic disease were used as controls $(n=7)$ ( $\triangleright$ Table 1$)$. There were no cases of suspected villous atrophy at the time of recruitment, but villous atrophy was pointed out in some cases as a result of the procedure.

\section{Pancreatic exocrine function (PEF) test}

The median (IQR) value of PT-PABA excretion rate, which was measured twice, was 54.4\% (46.52-76.88\%).

\section{Evaluation of small-intestinal villous blood flow based on endoscopic images}

In tests on 26 subjects, $4 \mathrm{NBI}$ magnified images were taken before application of $10 \%$ dextrose solution, and 6 NBI magnified images were taken every 30 seconds in the 3-minute interval after application of dextrose solution. In total, 268 images were taken, including 112 and 156 images taken before and after dextrose application, respectively. Evaluation of these 

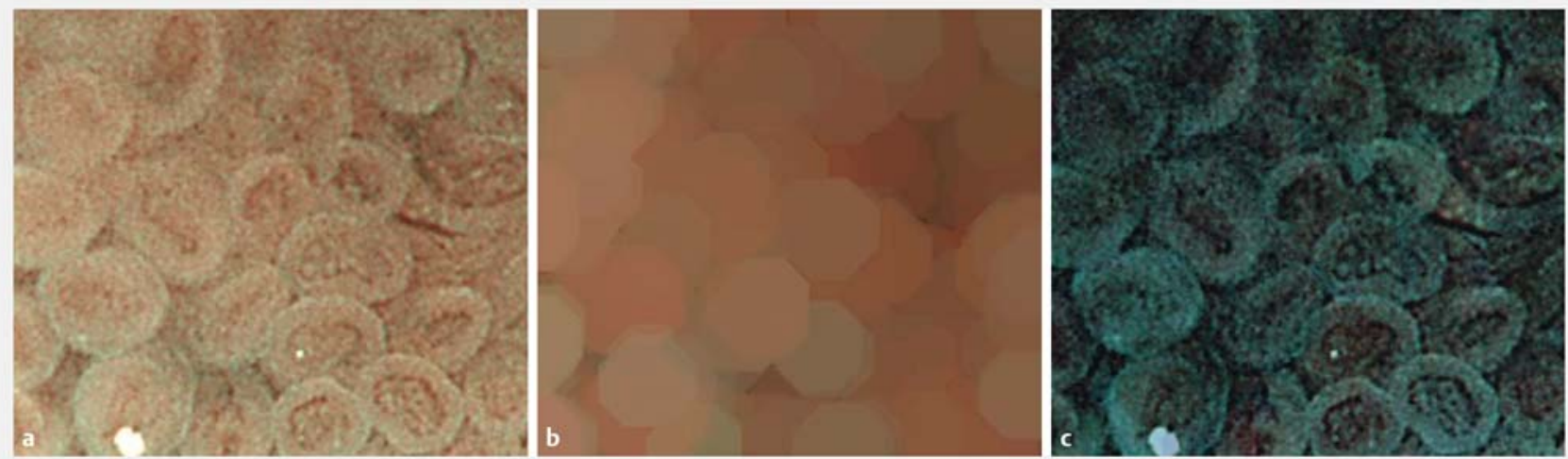

Fig. 5 a $1 / 4$ of the original image center was used to exclude the blurred part of the image. This is the same as the area of sharp lesion in - Fig. 4a. b A background image made by morphological opening processing. To standardize the brightness of each villus, we aimed to subtract the background brightness of each villus from the original image. Background brightness was obtained by smoothing the discontinuous pixel value within each villus. For smoothing, we used a morphological opening operation, which replaces each pixel value with the minimum value of its neighboring pixels. The morphological operation calls the range of the neighboring pixels "the structuring element". We wanted to set the structuring element, where the brightness was locally equalized, as the size of a single villus. Therefore, we first measured the radii of all villi manually in 20 randomly selected images with NBI obtained at maximum magnification, and the mean radius was found to be 32 pixels. Then we set the structuring element as a radius of 32 pixels using the "strel" function of MATLAB (MATLAB and Statistic Toolbox R2015b, The MathWorks Inc., Natick, MA, USA) [10,11]. With this setting, we performed the morphological opening operation using the "imopen" function of MATLAB $[10,11]$. By subtracting this background image from the original image, the brightness of the villi was standardized. c A corrected image. Subtraction of the background image ( $\nabla \mathbf{F i g . 5 b}$ ) from the original image ( $\triangleright$ Fig. $5 \mathbf{a}$ ) allowed the standardization of brightness of the villi.

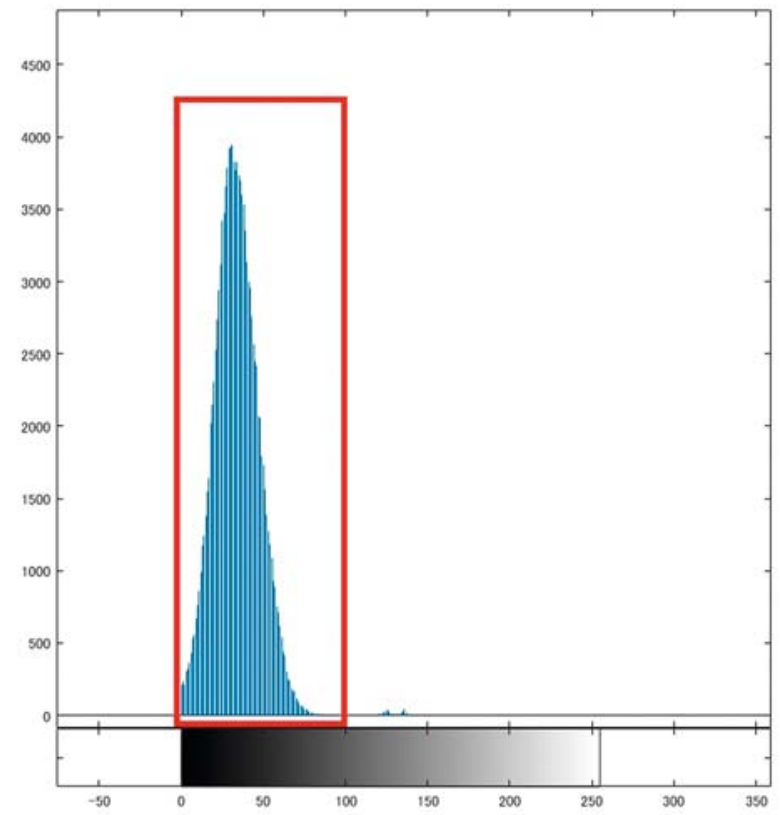

- Fig. 6 Histogram analysis was performed for the corrected images, in which the brightness of villi was standardized. Since the corrected images were color images, each pixel had 3 channel values: $R, G, B$. The values of these three channels were weighted and averaged for each pixel and converted into one luminance value. To exclude halation, pixels with luminance within the range 0 - 99 (square) were used to determine the standard deviation and defined as SDOV (Standard Deviation calculated from histogram made by luminance analysis Of Villi), because pixels with luminance $\geq 100$ represent halation. images gave median (IQR) values of VBFS and SDOV after dextrose application of $4(3-4)$ and $14.33(12.72-16.89)$, respectively. A comparison of SDOV and VBFS in each of the 268 images showed a significant positive correlation between these parameters $(P<0.0001, \rho=0.5882)$ ( $\vee$ Fig. 8$)$. This correlation shows that data from subjective evaluation are expressed well by objective data.

\section{Correlation between villous blood flow and PEF}

VBFS was examined as a potential index of PEF. VBFS and PEF had a significant positive correlation $(P=0.0013, \rho=0.5944)$ ( $\triangleright$ Fig.9). This result indicates that PEF can be evaluated using subjective evaluation based on still images taken after application of dextrose solution. The maximum value of SDOV in the 3minute interval after application of dextrose also showed a significant positive correlation with PEF $(P=0.0004, \rho=0.6421)$ ( $\triangleright$ Fig. 10). The correlation of PEF with SDOV (an objective index), was higher than that with VBFS (a subjective index based on the same endoscopic findings). This suggests that SDOV is appropriate as a new index for PEF.

\section{Discussion}

In the current study, a newly developed endoscope for the small intestine, SIF-Y0007, allowed further clarification of organ function correlation, since this endoscope has an $80 \times$ magnification function and improved insertability. This magnifying function enabled dynamic observation of the reaction of the small intestine to direct mucous membrane irritation by dispersion of nutrients. Dextrose was chosen as the nutrient because it is rapidly absorbed in the small intestine, and thus the appro- 


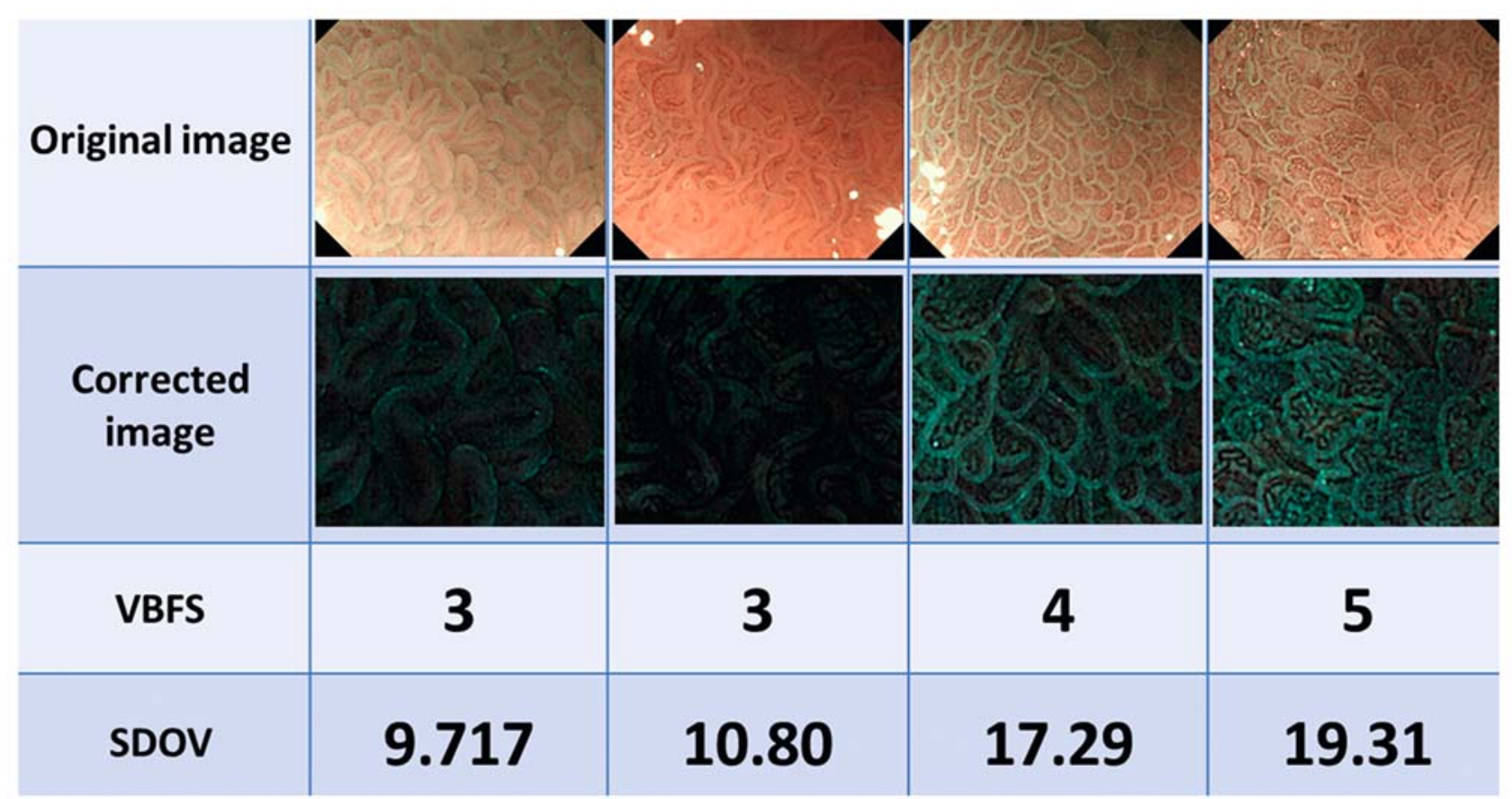

Fig. 7 Examples of luminance analysis. When blood flow increases in the villi, the number of pixels with low luminance will increase and show large variations. This results in an increased standard deviation (SD) obtained from the histogram. Therefore, SD can be used as an objective index of villous blood flow volume. We named the SD as SDOV: SD calculated from histogram made by luminance analysis Of Villi.

- Table 1 Patient demographics.

\begin{tabular}{|l|c|}
\hline Item & Value \\
\hline Age (range), y & $70.5(61.25-75.00)^{1}$ \\
\hline Sex, M:F & $21: 5$ \\
\hline Disease & \\
\hline - Chronic pancreatitis & 13 \\
\hline - Autoimmune pancreatitis & 5 \\
\hline - Pancreatic carcinoma & 1 \\
\hline - Pancreatic cystic disease & 4 \\
\hline - Others & 3 \\
\hline \begin{tabular}{l} 
PT-PABA excretion rate (\%) \\
\hline $\begin{array}{l}\text { VBFS (maximum value after spraying } \\
\text { dextrose solution) }\end{array}$
\end{tabular} & $54.4(46.52-76.88)^{1}$ \\
\hline $\begin{array}{l}\text { SDOV (maximum value after spraying } \\
\text { dextrose solution) }\end{array}$ & $16.92(14.12-19.40)^{1}$ \\
\hline $\begin{array}{l}\text { SDOV, Standard Deviation calculated from a histogram made by luminance } \\
\text { analysis Of Villi; VBFS, villous blood flow scale. } \\
\text { 1 Interquartile range (IQR). }\end{array}$ \\
\hline
\end{tabular}

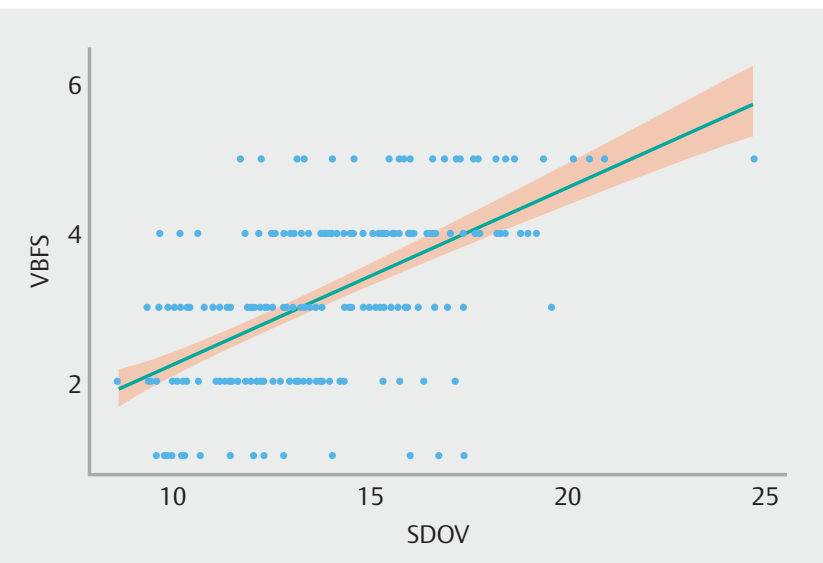

- Fig. 8 Correlation between VBFS and SDOV. A comparison of SDOV and VBFS in each of the 268 images showed a significant positive correlation between these parameters $(P<0.0001$, $\rho=0.5882$.

priate concentration can be determined. A $10 \%$ dextrose solution was ultimately used because the villous form could not be maintained due to the high osmotic pressure produced by a dextrose concentration of $20 \%$ or higher, causing difficulty in evaluating changes in internal blood flow.

In this study, we found a significant positive correlation between SDOV and PEF. Baumler et al. found that rats receiving total parenteral nutrition (TPN) can develop jejunum atrophy due to lack of nutrient irritation in the bowel [14]. In another 


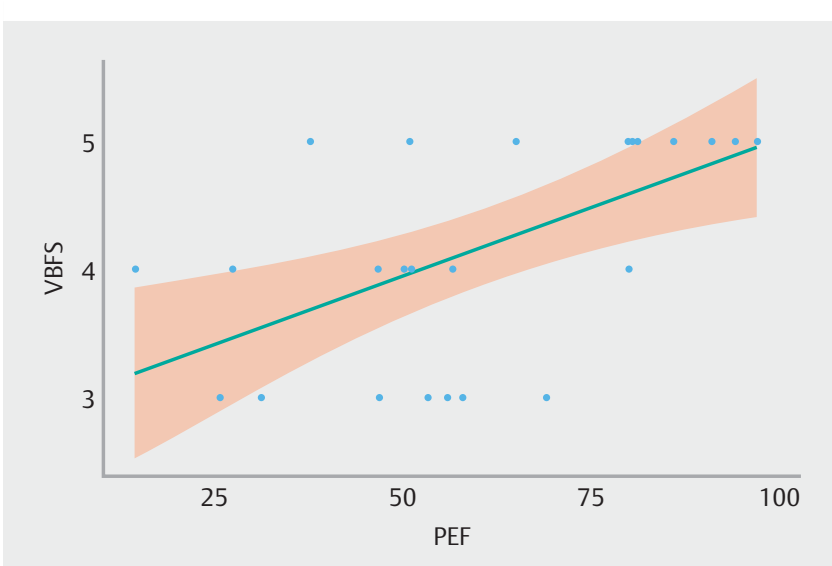

- Fig. 9 Correlation between VBFS and PEF. VBFS and PEF had a significant positive correlation $(P=0.0013, \rho=0.5944)$.

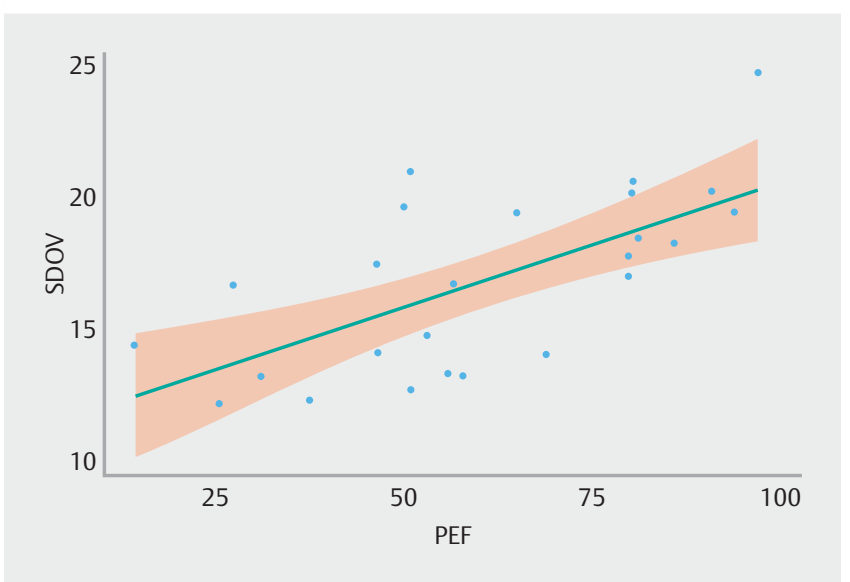

- Fig.10 Correlation between SDOV and PEF. The correlation of PEF with SDOV, which was based on an objective evaluation, was higher than that with VBFS, a subjective index based on the same endoscopic findings $(P=0.0004, \rho=0.6421)$. This suggests that SDOV is appropriate as a new index for PEF.

report, the same group suggested that oral and intravenous administration of an amino acid formulation improved decreased pancreatic function, but had no effects on atrophy of the jejunal mucous membrane in rats given TPN [15]. These reports suggest that, for villous function in the small intestine, explosive irritation by pancreatic enzymes may play an important role, rather than nutrient irritation of amino acids, and this may be the reason why SDOV reflects PEF.

In pancreatic diseases such as chronic pancreatitis and pancreatic cancer, both exocrine and endocrine insufficiency may develop, leading to malnutrition over time, and malnutrition worsens the prognosis of patients with these diseases [1,2]. Therefore, early detection of exocrine pancreatic insufficiency is important $[16,17]$. Evaluation of PEF based on SDOV is likely to be clinically useful, since in a PT-PABA excretion rate study, urine collection is required after internal administration of the agent, and this is difficult in clinical practice. The SDOV test can be performed after upper digestive tract screening using SIF-
Y0007, and can be completed in about 10 minutes, including magnified observation. The test is also relatively simple and non-invasive, compared to biopsy.

There are some limitations in this study. First, this is a retrospective, open label, single center study and included a small number of subjects. Second, the PT-PABA excretion rate, which we used for a PEF test, has a large margin of error; thus, our optical, dynamic assessment may also have a large margin of error. Endoscopic examination has been developed mainly for diagnosis of lesions, but the results of this study suggest that an objective functional diagnosis can also be made using endoscopy. Endoscopic evaluation of villous function in the small intestine after direct application of dextrose solution is likely to be useful in clinical practice and for clarification of the mechanisms underlying the functional correlation between the pancreas and small intestine.

\section{Competing interests}

None

\section{References}

[1] Bachmann J, Heiligensetzer M, Krakowski-Roosen $\mathrm{H}$ et al. Cachexia worsens prognosis in patients with resectable pancreatic cancer. J Gastrointest Surg 2008; 12: $1193-1201$

[2] Dewys WD, Begg C, Lavin PT et al. Prognostic effect of weight loss prior to chemotherapy in cancer patients. Eastern Cooperative Oncology Group. Am J Med 1980; 69: 491-497

[3] Altmann GG. Influence of bile and pancreatic secretions on the size of the intestinal villi in the rat. Am J Anat 1971; 132: 167-177

[4] Senegas-Balas F, Bastie MJ, Balas D et al. Histological variations of the duodenal mucosa in chronic human pancreatitis. Dig Dis Sci 1982; 27: 917-922

[5] Gubergrits NB, Linevskiy YV, Lukashevich GM et al. Morphological and functional alterations of small intestine in chronic pancreatitis. JOP 2012; 13: 519- 528

[6] Nakamura $\mathrm{Y}$, Itoh A, Kawashima $\mathrm{H}$ et al. Investigation of morphological and functional changes in the small intestine with pancreatic disease. Pancreas 2015; 44: $1352-1357$

[7] Hayashi D, Hirooka Y, Kawashima $\mathrm{H}$ et al. Functional correlation between the pancreas and the small intestine in humans: the first evaluation using a newly developed enteroscopy. Pancreas 2018; 47: $601-608$

[8] Shimosegawa T, Kataoka K, Kamisawa T et al. The revised Japanese clinical diagnostic criteria for chronic pancreatitis. J Gastroenterol 2010; 45: $584-591$

[9] Shimosegawa T, Chari ST, Frulloni L et al. International consensus diagnostic criteria for autoimmune pancreatitis: guidelines of the International Association of Pancreatology. Pancreas 2011; 40: 352 358

[10] Vincent L. Morphological grayscale reconstruction in image analysis: Applications and efficient algorithms. IEEE Trans Image Process 1993; 2: $176-201$

[11] Haralick RM, Sternberg SR, Zhuang X. Image analysis using mathematical morphology. IEEE Trans Pattern Anal Mach Intell 1987; PAMI-9: $532-550$ 
[12] Ihaka R, Gentleman R. R: a language for data analysis and graphics. J Comp Graph Stat 1996; 5: 299-314

[13] Wickham H. ggplot2: Elegant graphics for data analysis. New York: Springer-Verlag; 2009

[14] Baumler MD, Nelson DW, Ney DM et al. Loss of exocrine pancreatic stimulation during parenteral feeding suppresses digestive enzyme expression and induces Hsp70 expression. Am J Physiol Gastrointest Liver Physiol 2007; 292: G857-866
[15] Baumler MD, Koopmann MC, Thomas DD et al. Intravenous or luminal amino acids are insufficient to maintain pancreatic growth and digestive enzyme expression in the absence of intact dietary protein. Am J Physiol Gastrointest Liver Physiol 2010; 299: G338-347

[16] Rasmussen HH, Irtun O, Olesen SS et al. Nutrition in chronic pancreatitis. World J Gastroenterol 2013; 19: $7267-7275$

[17] Gilliland TM, Villafane-Ferriol N, Shah KP et al. Nutritional and metabolic derangements in pancreatic cancer and pancreatic resection. Nutrients 2017; 9: E243 